\title{
DPN-Generated Nanostructures as Positive Resists For Preparing Lithographic Masters or Hole Arrays
}

Khalid S. Salaita, Seung Woo Lee, David S. Ginger and Chad A. Mirkin

Department of Chemistry and International Institute for Nanotechnology, Northwestern

University, Evanston, IL 60208, USA

\section{Supporting Information}

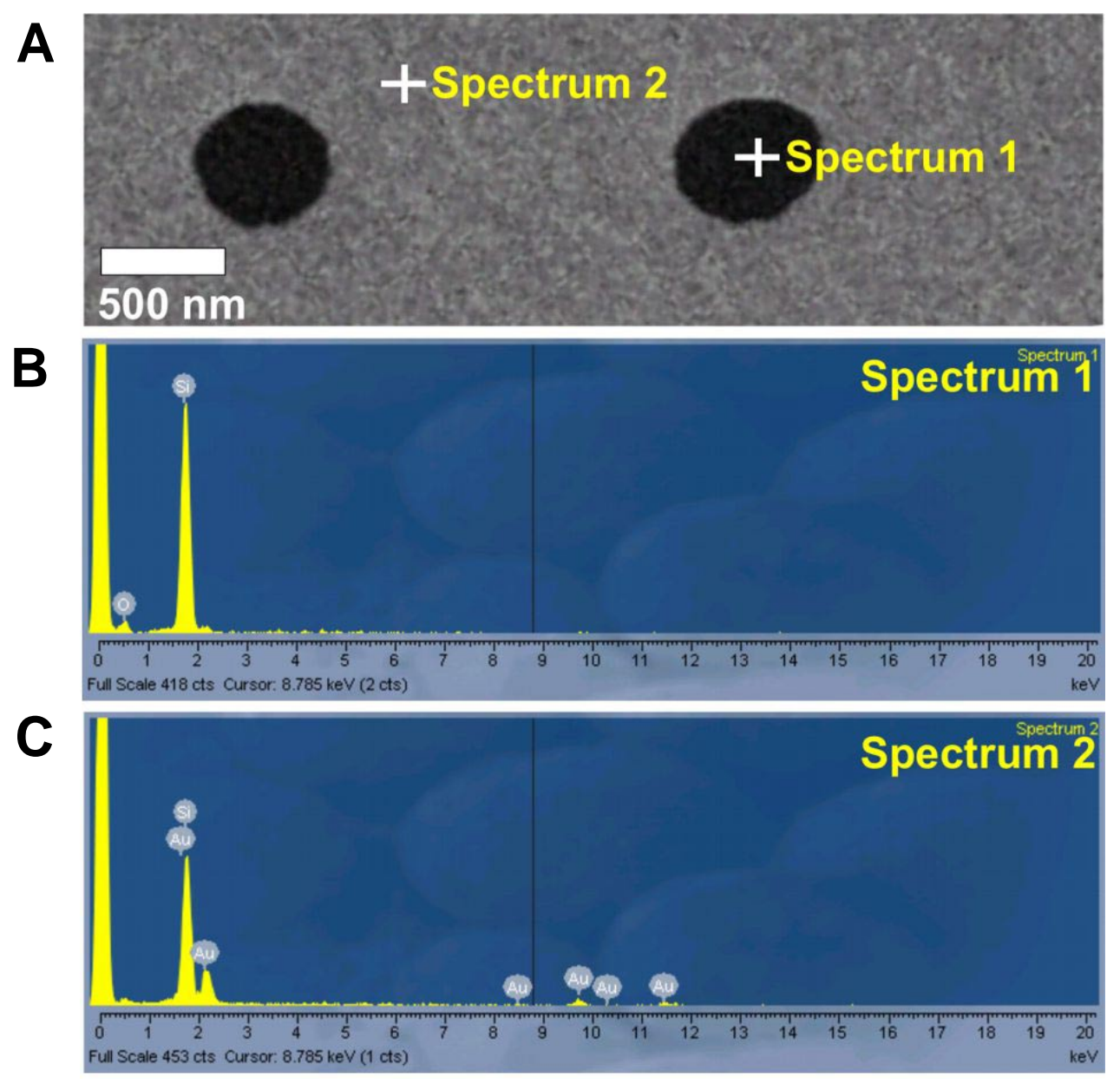

SI Figure 1. EDS analysis of DPN-templated hole structures in Au film. A) FESEM image of DPN-templated holes. B) and C) EDS analysis of highlighted regions "+" in 
SEM image, where spectrum 1 corresponds to hole structure and spectrum 2 corresponds to ODT passivated $\mathrm{Au}$. Note that spectrum 1 exclusively shows the elemental peaks associated with $\mathrm{SiO}_{x}$, and spectrum 2 exhibits the elemental peaks associated with both $\mathrm{Au}$ and $\mathrm{SiO}_{\mathrm{x}}$.
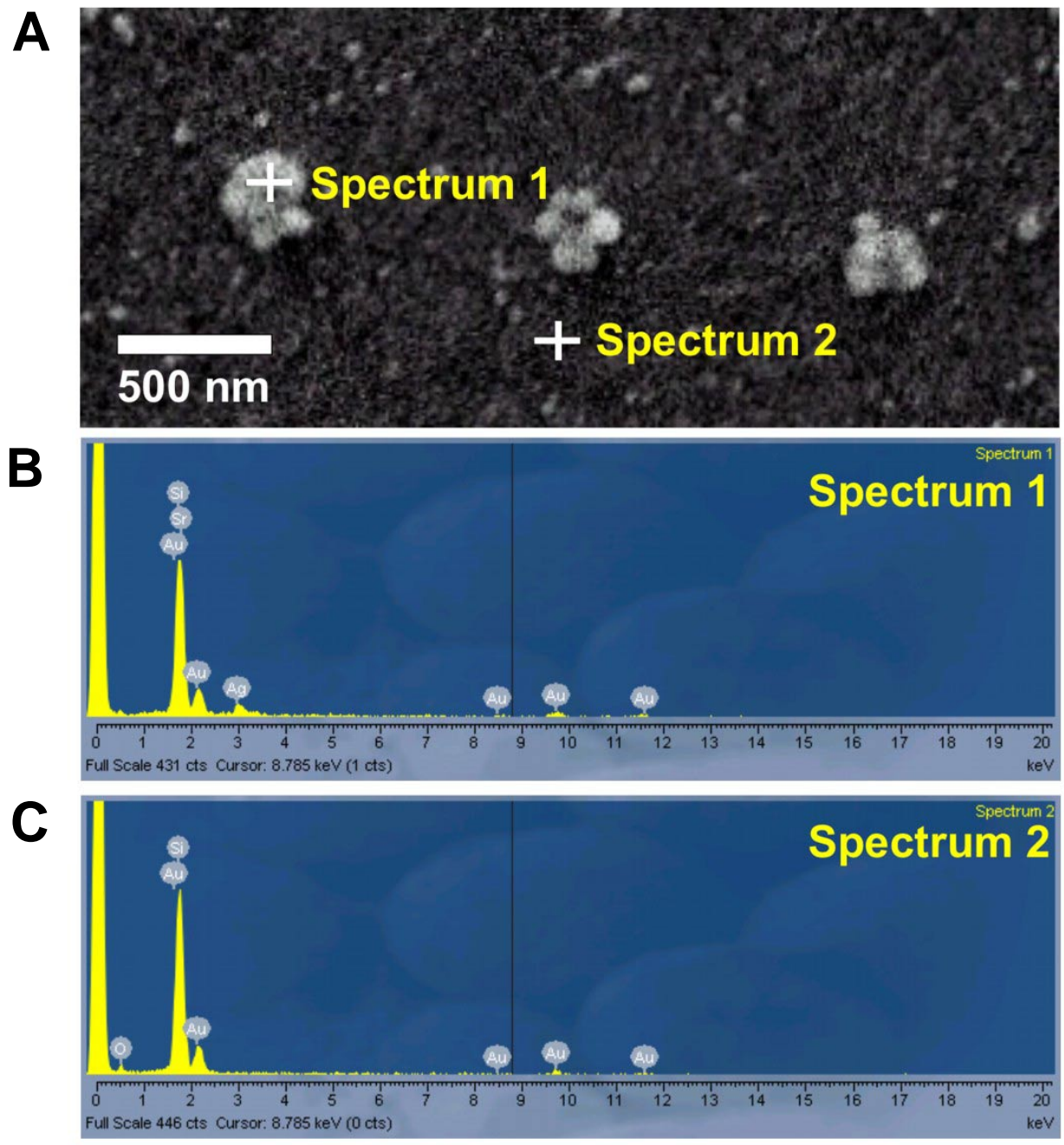

SI Figure 2. EDS analysis of DPN-templated Ag structures electrodeposited on $\mathrm{Au}$ film. A) FESEM image of DPN-defined Ag dot shaped structures. B) and C) EDS analysis of highlighted regions "+" in SEM, where spectrum 1 corresponds to an Ag structure and spectrum 2 corresponds to an ODT passivated Au region. Note that spectrum 1 exhibits the characteristic elemental signatures for both $\mathrm{Au}$ and $\mathrm{Ag}$, while spectrum 2 exhibits the characteristic elemental signatures for Au exclusively.

Dependence on the Applied Potential. Our previous work indicated that the bulk peak desorption potential of ODT and MHA SAMs differ by $\sim 100 \mathrm{mV}$ in $0.5 \mathrm{M} \mathrm{KOH}$ solution (Figure 2). The onset of electrochemical desorption for MHA perimeter sites was at -750 
$\mathrm{mV}$ (vs $\mathrm{Ag} / \mathrm{AgCl}$ in $0.5 \mathrm{M} \mathrm{KOH}$ ), whereas the onset of electrochemical desorption for ODT perimeter sites was at $-850 \mathrm{mV}$ (vs $\mathrm{Ag} / \mathrm{AgCl}$ in $0.5 \mathrm{M} \mathrm{KOH}) .{ }^{1,2}$ However, once the periphery of the MHA dots are blocked with ODT, electrochemical whittling no longer proceeds at $-750 \mathrm{mV}$ (SI Figure 3). Therefore, selective desorption of MHA over ODT does not proceed at potentials equal to or lower than $-750 \mathrm{mV}$. Conversely, if an overpotential of $-850 \mathrm{mV}$ is applied for $5 \mathrm{~min}$, desorption of the passivating ODT monolayer start to occur randomly across the substrate (SI Figure 4).
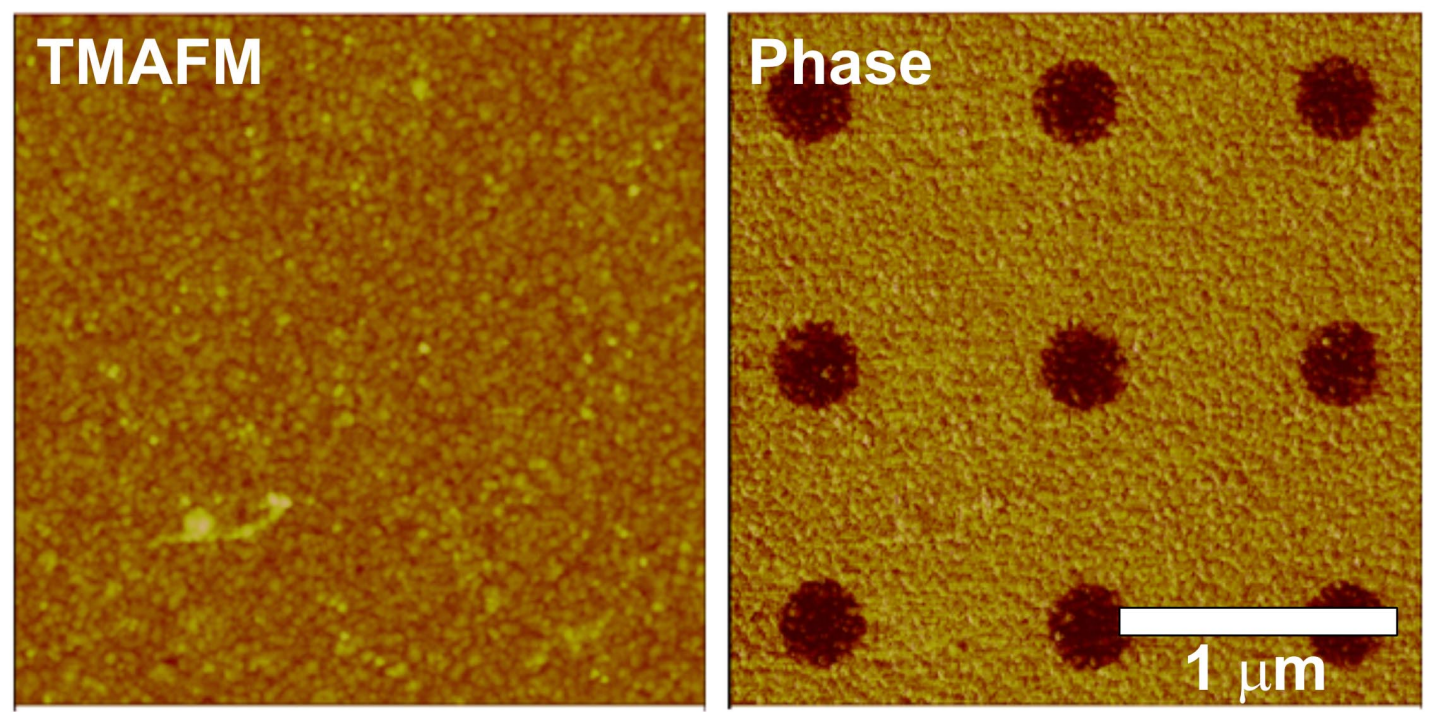

SI Figure 3. TMAFM and phase images of a $3 \times 3$ MHA dot array, where the background $\mathrm{Au}$ surface was passivated with ODT and a potential of $-750 \mathrm{mV}$ was applied for $5 \mathrm{~min}$. The sample was then exposed to $1 \mathrm{mM} \mathrm{KCN}$ for $20 \mathrm{~min}$. Note that the MHA structures remain intact and the Au substrate is not etched. $\mathrm{Z}$ scale is $20 \mathrm{~nm}$. 


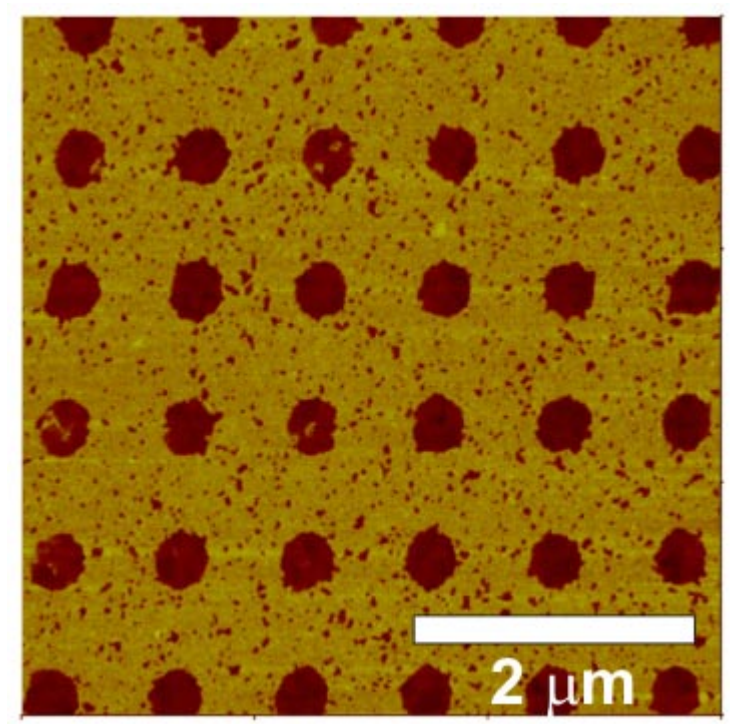

SI Figure 4. TMAFM image of a hole array generated by applying a potential of -850 $\mathrm{mV}$ for $5 \mathrm{~min}$, and subsequently exposing the substrate to an alkaline $\mathrm{CN}^{-}$containing solution at OCP. Note the presence of small pits in the ODT passivating layer. Z scale is $60 \mathrm{~nm}$.

Dependence on the Desorption Time. Another important parameter that controls the formation of holes is the duration of applied desorption potential. SI Figure 5 demonstrates this dependence by showing three different MHA patterned Au substrates where all the conditions were maintained $\left(E_{\mathrm{des}}=-800 \mathrm{mV}\right)$ except for the length of time that the desorption potential was applied. When the desorption pulse is applied for longer periods of time $(\mathrm{t}=8 \mathrm{~min})$, some desorption of the passivating ODT monolayer occurs, and subsequently the Au surface is pitted (SI Figure 5B). If a desorption pulse is not applied $(\mathrm{t}=0 \mathrm{~min})$, then both the MHA and ODT structures remain intact, and consequently the Au film is protected (SI Figure 5C). This indicates that in order to minimize damage to the passivating ODT layer it is critical to minimize the length of time that the desorption potential is applied. 

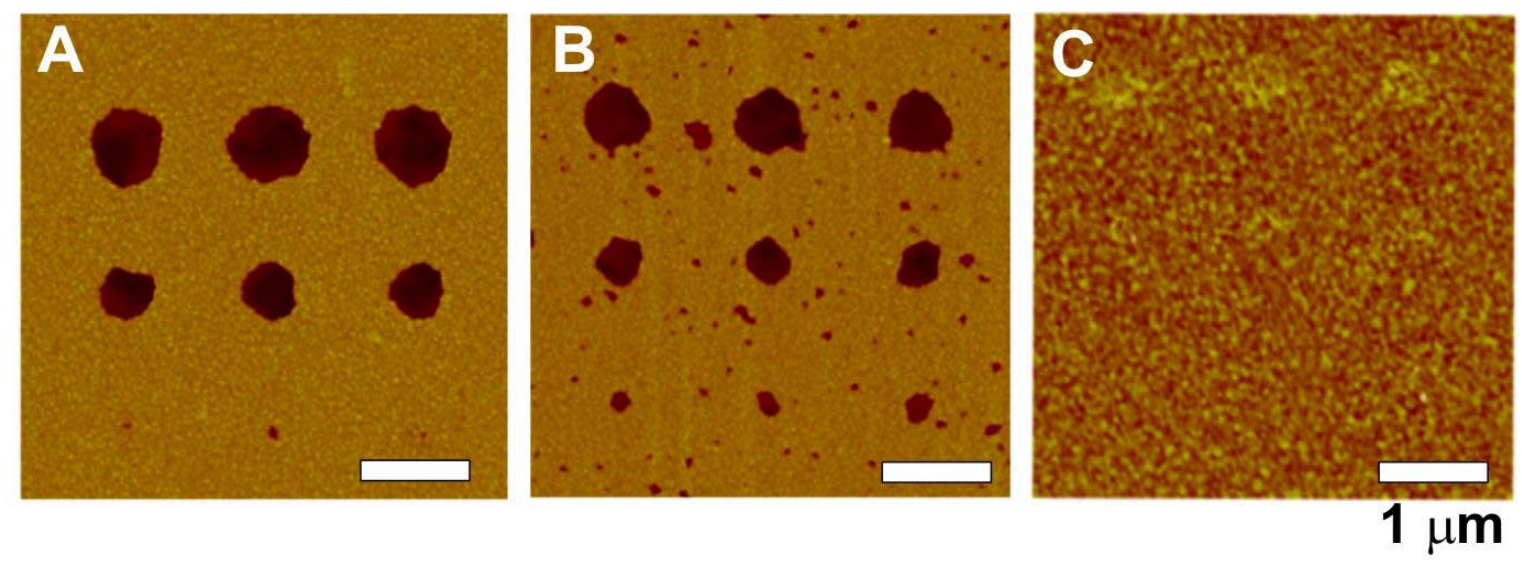

SI Figure 5. TMAFM images of $3 \times 3$ hole arrays generated by applying a selective desorption potential of $-800 \mathrm{mV}$ for 5,8 , and 0 min which correspond to $\mathrm{A}, \mathrm{B}$ and $\mathrm{C}$, respectively. $\mathrm{Z}$ scale is $80 \mathrm{~nm}$ for $\mathrm{A}$ and $\mathrm{B}, 10 \mathrm{~nm}$ for $\mathrm{C}$.

Hole Arrays on Transparent Substrates. In order to measure the transmission spectra of the DPN-generated hole arrays it is necessary to use a transparent substrate. Therefore, the selective etching of MHA patterned templates was performed on Au films supported on quartz substrates in place of silicon substrates. The quality of hole arrays generated on quartz was comparable to those on native-oxide coated silicon (SI Figure 6), although the silicon substrates contained a smaller density of dust and defects. The transmission mode micrograph of the triangular hole arrays appeared to have a slight bluish-green color, which indicated that the nanostructured Au film interacts with transmitted light. 

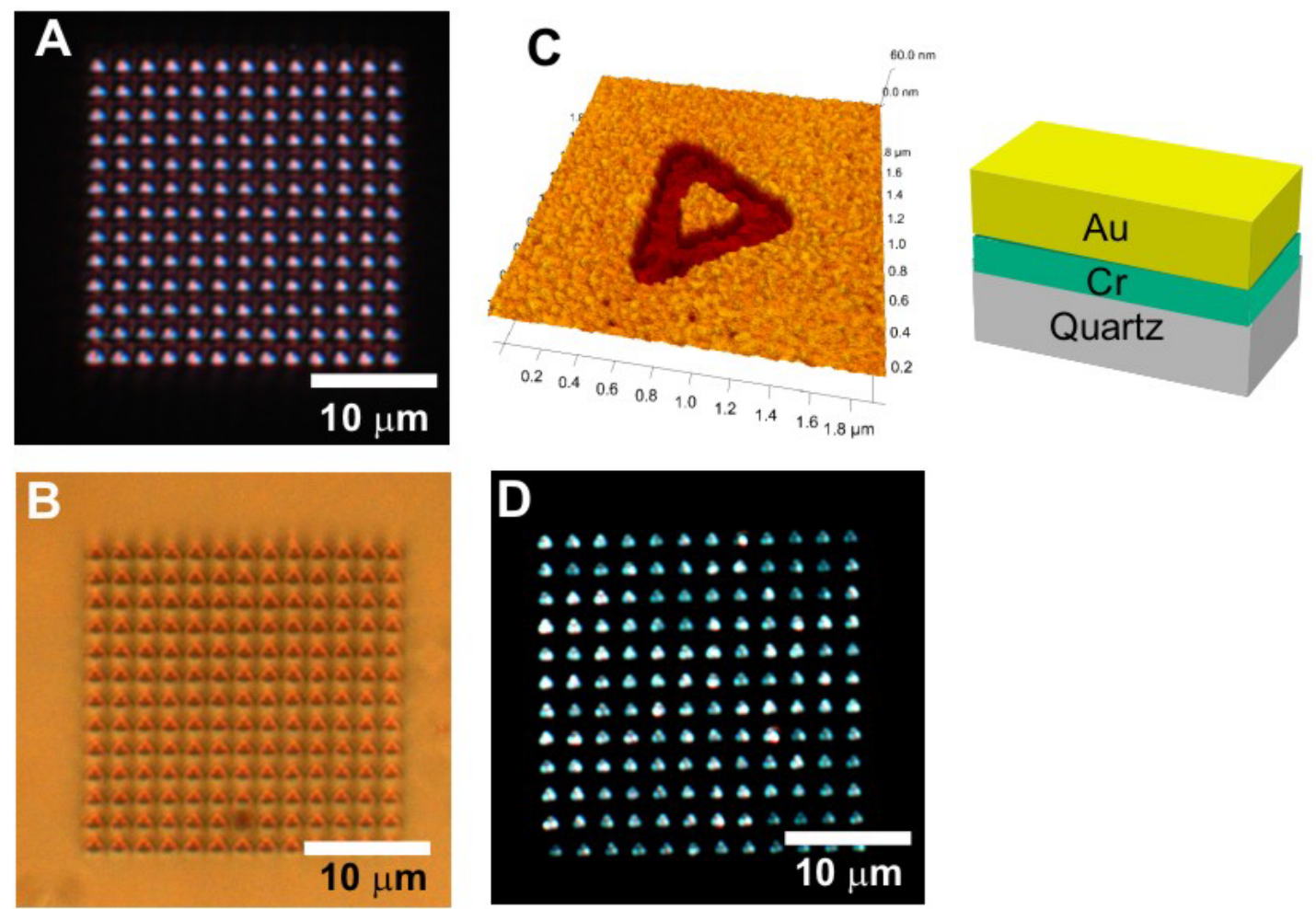

SI Figure 6. Measuring the optical properties of nanostructured Au films supported on a quartz substrate (scheme right). Optical micrographs of triangular hole array in A) darkfield reflection mode, B) brightfield reflection mode, D) brightfield transmission mode. C) TMAFM image of a representative triangular structure etched in a $\mathrm{Au}$ film.

\section{Experimental Section}

Chemicals. 1-octadecanethiol (ODT) (98\%), 16-mercaptohexadecanoic acid (MHA) (90\%), KCN (97\%), KOH (semiconductor-grade) were purchased from Aldrich Chemical Co. Ethanol (ACS/USP grade) was purchased from Pharmcoproducts Inc. Acetonitrile (reagent grade) and methylene chloride (99.9\%) were purchased from Fisher Scientific. All chemicals were used as received.

Substrates, Patterning, and Imaging. In a typical experiment, Au-coated (20-60nm), Ti-coated $(5 \mathrm{~nm})$ silicon oxide $\left(\mathrm{Au} / \mathrm{Ti} / \mathrm{SiO}_{\mathrm{x}} / \mathrm{Si}\right)^{3}$ was patterned via DPN with MHA using an Atomic Force Microscope (AFM, CP, Veeco/ThermoMicroscopes, Sunnyvale, CA) equipped with a $100-\mu \mathrm{m}$ scanner with closed-loop scan control, or an Nscriptor ${ }^{\mathrm{TM}}$ (Nanoink Inc., Chicago, IL) equipped with a 100- $\mu \mathrm{m}$ scanner and closed-loop scan 
control and commercial lithography software (DPNWrite ${ }^{\mathrm{TM}}$, DPN System-1, Nanoink Inc., Chicago, IL). Gold-coated $\mathrm{Si}_{3} \mathrm{~N}_{4}$ AFM cantilevers (Microlever, Veeco/ThermoMicroscopes, Sunnyvale, CA) with a spring constant of $0.05 \mathrm{~N} / \mathrm{m}$ and commercially available gold-coated $\mathrm{Si}_{3} \mathrm{~N}_{4}$ multi-cantilever A-26 arrays with a spring constant of $0.097 \mathrm{~N} / \mathrm{m}$ (NanoInk, Chicago, IL) were used for patterning. MHA-coated tips were prepared by immersing the cantilevers in an acetonitrile solution saturated with MHA for a few seconds. They were washed with neat ethanol solution and subsequently blown dry under a stream of $\mathrm{N}_{2}$.

Imaging of DPN-generated patterns was performed using a clean AFM tip under conditions identical to those used for patterning in contact mode. Patterned structures, post etching, were characterized by SEM (Leo Gemini 1525) and dark field microscopy (Zeiss Axiovert 100A inverted microscope, Thornwood, NY). The microscope was equipped with a Penguin 600CL digital camera and StreamPix software. Tapping mode AFM (TMAFM) images were collected with a Nanoman AFM equipped with a Nanoscope IV controller from Veeco (Santa Barbara, CA) with silicon tips (NCH-W, Veeco, spring constant $40 \mathrm{~N} / \mathrm{m}$ ) scanned at a rate of $0.5 \mathrm{~Hz}$ and set to a pixel resolution of $512 \times 512$.

Selective Desorption. To effect selective electrochemical desorption of MHA, the substrate with prepatterned MHA features was passivated in an ethanolic $5 \mathrm{mM}$ ODT solution for 1 hour, and then placed into an electrochemical cell and used as the working electrode in a three-electrode configuration (Bioanalytical System potentiostat, BAS 100B). The counter electrode was a freshly flamed Pt wire, and all potentials were recorded with a $\mathrm{Ag} / \mathrm{AgCl}(3 \mathrm{M} \mathrm{NaCl})$ as the reference electrode. Following selective electrochemical desorption, the substrate was washed with Nanopure ${ }^{\mathrm{TM}}$ water and ethanol 
respectively, and then dried under a flow of $\mathrm{N}_{2}$. The substrate was immediately used for further experiments.

Etching. $\mathrm{CN}^{-}$-induced etching of substrates under potential control was performed in an electrochemical cell as described above, where the electrolyte was an alkaline solution containing KCN. ${ }^{4}$ The open-circuit potential (OCP) of an ODT-passivated Au substrate was about $-520 \mathrm{mV}$ (vs $\mathrm{Ag} / \mathrm{AgCl}$ ). The $\mathrm{OCP}$ was sufficiently positive to initiate dissolution of unprotected $\mathrm{Au}$, and patterned substrates were immersed in the etching solution without stirring or mixing, and subsequently washed with Nanopure ${ }^{\mathrm{TM}}$ water and ethanol.

Electroplating. Silver cyanide/potassium cyanide plating solution (Technic Silver 1025) and potassium gold cyanide plating solution (Orotemp 24 plating solution) were purchased from Technic Inc., Cranston, RI (www.technic.com). Prior to use, solutions were passed through a syringe filter with a pore size of $0.2 \mu \mathrm{m}$. Deposition was performed at ambient conditions under potentiostatic control (typically $-800 \mathrm{mV}$ vs $\mathrm{Ag} / \mathrm{AgCl})$ while stirring mildly $(\sim 100 \mathrm{rpm})$. Substrates were washed with Nanopure ${ }^{\mathrm{TM}}$ water and ethanol respectively after deposition.

PDMS replica. Stamps were fabricated by placing a DPN-prepared master (by using the procedure describe above) in a glass Petra dish, followed by pouring over the master a mixture of polydimethlysiloxane (PDMS, Sylgard 184, Dow Corning, Midland, MI) in the ratio of 10:1 (v:v) monomer to initiator. After one hour degassing the elastomer was cured for $2 \mathrm{~h}$ at $60{ }^{\circ} \mathrm{C}$, and then gently peeled from the master and inspected by optical microscopy.

\section{References:}


(1) Zhang, Y.; Salaita, K.; Lim, J. H.; Lee, K. B.; Mirkin, C. A. A Massively Parallel Electrochemical Approach to the Miniaturization of Organic Micro- and Nanostructures on Surfaces. Langmuir 2004, $20,962$.

(2) Zhang, Y.; Salaita, K.; Lim, J. H.; Mirkin, C. A. Electrochemical whittling of organic nanostructures. Nano Lett 2002, 2, 1389.

(3) Weinberger, D. A.; Hong, S. G.; Mirkin, C. A.; Wessels, B. W.; Higgins, T. B. Combinatorial generation and analysis of nanometer- and micrometer-scale silicon features via "dip-pen" nanolithography and wet chemical etching. Adv Mater 2000, 12, 1600.

(4) Zamborini, F. P.; Crooks, R. M. In-situ electrochemical scanning tunneling microscopy (ECSTM) study of cyanide-induced corrosion of naked and hexadecyl mercaptan-passivated Au(111). Langmuir 1997, 13, 122. 